\title{
Screening of CXC chemokines in the microenvironment of ovarian cancer and the biological function of CXCL10
}

\author{
Weiyuan $\mathrm{Li}^{\dagger}$, Ji-Ao Ma ${ }^{\dagger}$, Xun Sheng and Chunjie Xiao ${ }^{*}$
}

\begin{abstract}
Background: This study aims to screen and identify the biological functions and prognostic value of CXC chemokines in ovarian cancer (OC) through bioinformatics and molecular biology methods, and to provide data support for the selection of biomarkers and prognostic analysis of OC.

Methods: In this study, GEO, ONCOMINE, GEPIA, cBioPortal, GeneMANIA, Metascape, STRING, TRRUST, and TIMER databases were used to study CXC chemokines. Angiogenesis and T cell killing assay were used to detect the effect of CXCL10 on tumor cell immunity and angiogenesis. Real-time quantitative PCR (qRT-PCR), immunoblotting, and ectopic tumor formation experiments were used to verify the effect of CXCL10 on ovarian cancer tumors.

Results: We found that CXCL1, CXCL10, CXCL11, CXCL13, and CXCL14 were significantly upregulated in OC samples compared with normal tissues. Our data showed that there was a relationship between the expression of CXC chemokines and the infiltration of six types of immune cells significant correlation. In vitro assay confirmed that overexpression of CXCL10 could enhance the killing effect of T cells and inhibit angiogenesis. Further in vivo assay had shown that CXCL10 could affect the progression of ovarian cancer by increasing the expression of cytotoxic T cells and inhibiting angiogenesis.
\end{abstract}

Conclusion: In conclusion, we hope that our data will provide new insights into the development of immunotherapy and the selection of prognostic markers for patients with OC.

Keywords: Ovarian cancer, Chemokines, Angiogenesis, Immune infiltration, Bioinformatics

\section{Background}

Ovarian cancer $(\mathrm{OC})$ is the sixth most common female cancer in the world, the second largest gynecological malignant tumor, and the deadliest tumor of the human female reproductive system [1]. Although treatments for ovarian cancer had improved, most patients are still at risk of recurrence. In recent years, there was evidence that $\mathrm{OC}$ was an immunogenic tumor, and it had been observed that tumor-infiltrating lymphocyte subsets

*Correspondence: chjxiao@ynu.edu.cn

tWeiyuan Li and Ji-Ao Ma contributed equally to this work. School of Medicine, Yunnan University, No.2, Cuihu North Road, Kunming 650091, Yunnan Province, People's Republic of China were closely related to the overall survival of patients [2]. With the increasing research of immunotherapy in the treatment of tumors, its efficacy has attracted people's attention. However, the current specific mechanism is still unclear. Therefore, this study attempts to screen and verify more therapeutic targets and prognostic biomarkers through a combination of bioinformatics and molecular biology.

Chemokines were secreted by tumor cells and other types of cells and regulate anti-tumor immune responses by regulating immune cell transport and other pathways [3]. Current evidence suggests that chemokines could directly and indirectly modulates immunity and modulates tumor immunology and biological phenotypes, original author(s) and the source, provide a link to the Creative Commons licence, and indicate if changes were made. The images or other third party material in this article are included in the article's Creative Commons licence, unless indicated otherwise in a credit line to the material. If material is not included in the article's Creative Commons licence and your intended use is not permitted by statutory regulation or exceeds the permitted use, you will need to obtain permission directly from the copyright holder. To view a copy of this licence, visit http://creativecommons.org/licenses/by/4.0/. The Creative Commons Public Domain Dedication waiver (http://creativeco mmons.org/publicdomain/zero/1.0/) applies to the data made available in this article, unless otherwise stated in a credit line to the data. 
thereby affecting angiogenesis, tumor proliferation, metastasis, and patient prognosis [4]. CXC chemokine was an important role of the chemokine family, so it may be a potential therapeutic target and prognostic biomarker for a variety of cancers including OC.

Studies had determined the expression and function of some CXC chemokines in OC, but the current research is still very scarce $[5,6]$. In addition, the identification of effective molecules as therapeutic targets and biomarkers of OC still urgently needs our in-depth discussion. Therefore, we conducted comprehensive bioinformatics analysis on the expression and function of CXC chemokines in OC through a variety of databases. Through screening, we found that the differential expression of CXCL10 was the most significant. In vitro and in vivo experiments were used to further verify the role of CXCL10 in OC immunity and angiogenesis. Through the discussion of this study, we try to provide more powerful data support for clinicians to choose appropriate drugs and more accurate prognostic information in the treatment of $\mathrm{OC}$ patients.

\section{Materials and methods}

\section{Gene Expression Omnibus analysis}

The data set used is from Gene Expression Omnibus (GEO) database, and the download data format is MINIML (GSE6008 and GSE66957). Box plots are drawn by boxplot; PCA graphs are drawn by R software package ggord. The box plot is implemented by the $\mathrm{R}$ software package ggplot2; the heat map is displayed by the $\mathrm{R}$ software package pheatmap.

\section{ONCOMINE}

In this study, ONCOMINE (www.oncomine.org) extracted data to evaluate the level of CXC chemokines in OC [7]. The significance threshold is $P<0.05$, fold change $>2$, and the gene ranking is the top $10 \%$.

\section{Gene expression profiling interactive analysis}

In this study, Gene expression profiling interactive analysis (GEPIA)'s "single gene analysis" module was used to analyze the differences in mRNA expression and pathological stages between tumor and normal tissues, as well as the prognostic analysis of CXC chemokines. Use the "Ovarian serous cystadenocarcinoma (OV)" data set. The Kaplan-Meier curve was used for prognostic analysis.

\section{cBioPortal for cancer genomics}

In this study, the cBioPortal (www.cbioportal.org) database was used to analyze the gene changes and co-expression modules of CXC chemokines obtained from TCGA data sources [8]. The OV specimens (TCGA) of 606 cases were analyzed.

\section{GeneMANIA}

We use GeneMANIA (http://www.genemania.org) to analyze CXC chemokine protein and genetic interaction information, co-expression and similarity of protein domains to the submitted genes [9].

\section{STRING}

STRING: functional protein association networks (https://string-db.org/). We performed PPI network analysis on CXC chemokines with different expressions to explore their interactions.

\section{Metascape}

In this study, Metascape databases was used to perform gene ontology (GO) and Kyoto Encyclopedia of Genes and Genome (KEGG) pathway enrichment analysis on 50 genes related to CXC chemokines [10].

\section{TRRUST}

The trust (https://www.grnpedia.org/trrust/) database contains 8444 transcription factor (TF) target regulatory relationships of 800 human transcription factors [11]. We use this data to predict the transcription factors of CXC chemokines.

\section{Immune infiltration analysis}

We use the TIMER (https://cistrome.shinyapps.io/ timer/) platform for immune infiltration analysis [12]. In this study, the "gene module" and "survival module" were used to evaluate the correlation between CXC chemokine levels and immune cell infiltration and the correlation between clinical results and immune cell infiltration and CXC chemokine expression.

\section{Cell culture}

SK-OV-3 and VE cells were obtained from Procell company (Wuhan, China). All cell lines were cultured in DMEM medium containing 10\% FBS and 1\% double antibodies. All the cells were incubated at the condition of 37 ${ }^{\circ} \mathrm{C}, 5 \% \mathrm{CO}_{2}$.

\section{Plasmid construction and cell transfection}

On the GenePharma (Shanghai, China), the transfected materials si-CXCL10 and CXCL10 were purchased. Twenty-four hours before transfection, SK-OV-3 cell in the exponential phase were digested by pancreatin and made into cell suspension. Si-CXCL10 and CXCL10 were used for the in vitro assay. After trypsinization from flasks, cells were cultured in six-pore plates, incubated at $37{ }^{\circ} \mathrm{C}$ with $5 \% \mathrm{CO}_{2}$ for $18-24 \mathrm{~h}$. Three hours before transfection, cells at about $80-90 \%$ confluency were changed to the serum and antibiotic-free media. Then, cells were transfected using Lipofectamin 2000 reagent 
(Life Technologies, Gaithersburg, MD, USA) referring to manufacturer's instructions and incubated at the same conditions as above for $48 \mathrm{~h}$. In addition to the in vivo experiment, based on the manual, concentrated lentiviral solutions of CXCL10 were mixed with two nutrient solution contains ID8 cell, respectively. Finally, the cells were digested by pancreatin and injected into mice after incubation for $48 \mathrm{~h}$.

\section{T cell-mediated cells killing assay}

Refer to reports [13], through the CCK8 test and apoptosis assay to test the $\mathrm{T}$ cell killing assay. Then SK-OV-3 cells and T cells were co-cultured for $24 \mathrm{~h}$, and then used for related treatment. The cell viability was analyzed by a microplate reader at $450 \mathrm{~nm}$.

\section{Cell proliferation ability test}

For CCK-8 assay, we employed Cell Counting Kit (Yeasen, China) to determine the viability of cells. The optical absorbance was measured at $450 \mathrm{~nm}$.

\section{Flow cytometry}

The treated cells $(1 \times 106)$ were seeded in 6-well plates a. After $48 \mathrm{~h}$ of treatment, cells were harvested and centrifuged to remove the supernatant. To perform apoptosis assays, cells were resuspended in binding buffer and stained with Annexin V and PI (BestBio, China). Finally, a flow cytometer (Beckman Coulter, USA) was used to assess cell apoptosis.

\section{Angiogenesis assay}

In this study, $50 \mu \mathrm{L} /$ well of Matrigel Matrigel was added to a 96-well plate, avoiding air bubbles when added, and placed in a cell incubator for $60 \mathrm{~min}$ to solidify, and 100 $\mu \mathrm{L}$ of cell suspension was added to each well (the tumor cell culture medium was treated with different treatments). Then we put it into a cell incubator for routine culture after making the mark, and take a photo with a microscope after incubating for $24 \mathrm{~h}$. The tube formation was visualized under an inverted microscope. Enclosed networks of tube structures from three randomly chosen fields were recorded under a Nikon microscope.

\section{Animal and tumor models}

$16 \mathrm{BALB} / \mathrm{c}$ mice aged 3-5 weeks were purchased from Charles river Laboratories (Beijing, China). All animals are kept in a constant temperature, constant humidity and no specific pathogen level animal center. Guarantee their unlimited water and diet. All animal studies were approved by the Animal Ethical and Welfare Committee of Yunnan University. The animals were randomly divided into 2 groups (NC group, CXCL10 group, $n=$ 6) and subcutaneously inoculated in the right flank with
$5 \times 10^{5}$ ID8 cells. Tumor volume was calculated using the formula: $\pi / 6 \times$ length $\times$ width 2 . The tumor size was measured every 3 days. On day 30, the mice were killed, and the tumors were dissected and weighed.

\section{Real-time PCR}

Total RNA was extracted by the TRIzol (Ambion, USA). All qRT-PCR processes were accomplished using the SYBR Green qPCR Master Mix (MedChem Express, NJ, USA). The results were calculated by the $2^{-\Delta \Delta \mathrm{Ct}}$ method. GAPDH was used for normalization as a control. Primers were shown in Supplementary Table 1.

\section{Hematoxylin-eosin, Ki67, and TUNEL staining}

In this study, the tumor tissues of mice in each treatment group were paraffin-embedded and sliced, and then the tissues were stained with the Ki-67 immunohistochemistry kit and Tunel analysis kit referring to the manufacturer's method. Hematoxylin and eosin were used to stain paraffin sections of mouse tumor tissues after deparaffinization and dehydration. Finally, observe the cell morphology under a microscope. We used ImageJ software to quantify the number of KI67, TUNEL and CD8-positive cells.

\section{Immunohistochemistry and immunofluorescence}

The immunohistochemistry (IHC)-stained tissue sections were reviewed under microscope by 3 pathologists who were blinded to the clinical parameters, and scored independently according to the intensity of cellular staining and the proportion of stained tumor cells. The VEGFA and COX2 proteins were immunohistochemically stained yellowish to brown in the cytoplasm, and displayed all or none mode in tumor tissues. The degree of immunostaining of indicated proteins was evaluated and scored by 3 independent observers. Briefly, each sample was scored according to staining intensity (no staining $=0$; weak staining $=1$; moderate staining $=2$; strong staining $=3$ ) and the number of stained cells ( $0 \%$ $=0 ; 1-25 \%=1 ; 26-50 \%=2 ; \geq 51 \%=3)$. The staining index (SI) was calculated as the product of staining intensity $\times$ percentage of positive tumor cells, result in scores of $0,1,2,3,4,6$, and 9 . The microvessel density (MVD) was evaluated by CD34 immunofluorescence staining. MVD was calculated as the average count of microvessel in the 4 hot spots at high magnification $(200 \times)$.

\section{Western blot}

First, we extract proteins from cells and tissues in different treatment groups, and then quantify each group of proteins with the BCA kit. Secondly, after electrophoresis of different groups of proteins and transfer to the membrane, the primary antibodies of human and 


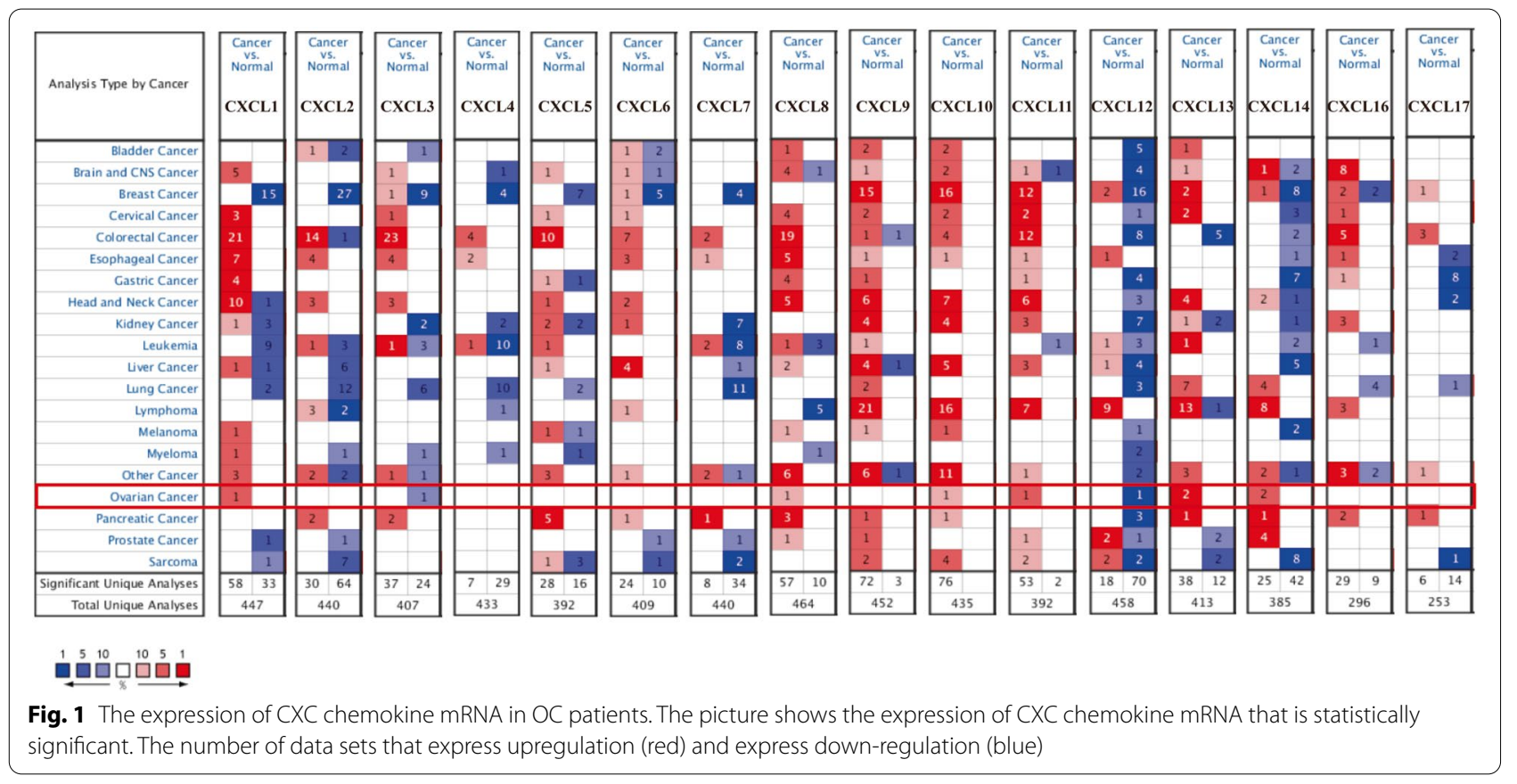

mouse VEGFA, CD34, COX2, and GAPDH (Abcam) were incubated in the membrane overnight at $4{ }^{\circ} \mathrm{C}$ and then combined with the secondary antibody. Finally, use an enhanced chemiluminescence kit to check the binding of the antibody.

\section{Statistical analysis}

All data in this study used Excel for data database construction and input, and SPSS 22.0 and GraphPad prism 9 software for data statistics. The measurement data were expressed in the form of mean \pm SEM, the comparison between multiple groups is compared by one-way analysis of variance (ANOVA), and the Tukey's was used for pairwise comparison. Inspection level $\alpha=$ 0.05 .

\section{Result}

The level of CXC chemokines expression in $O C$ patients

Transcriptional levels of 16 CXC chemokines (excluding CXCL15) in OC and ovarian tissues were analyzed using Oncomine database. It was found that the transcriptional levels of CXCL1, CXCL8, CXCL10, CXCL11, CXCL13, and CXCL14 in OC tissues were significantly increased. The transcription levels of CXCL3 and CXCL12 were lower than those in normal tissues (Fig. 1 and Supplementary Fig. 1). In addition, we have also demonstrated this phenomenon in a large body of literature (Table 1) [14-17, 20, 21]. As shown in Table 1, among the upregulated CXC chemokines, CXCL10 had the highest differential expression multiple, and similarly, CXCL12 had the highest differential expression multiple when downregulated.

Table 1 The mRNA levels of CXC chemokines in different types of OC tissues and normal tissues at transcriptome level (ONCOMINE)

\begin{tabular}{|c|c|c|c|c|c|}
\hline TLR & Type & Fold change & $t$ test & $P$ value & References \\
\hline CXCL1 & Ovarian Serous Adenocarcinoma & 2.753 & 4.093 & 0.004 & [14] \\
\hline CXCL3 & Ovarian Serous Adenocarcinoma & -5.279 & -4.912 & 0.000 & [15] \\
\hline CXCL8 & Ovarian Serous Adenocarcinoma & 2.455 & 2.562 & 0.015 & [16] \\
\hline CXCL10 & Ovarian Serous Adenocarcinoma & 9.290 & 5.697 & 0.000 & [17] \\
\hline CXCL11 & Ovarian Serous Adenocarcinoma & 2.995 & 9.548 & 0.000 & TCGA \\
\hline CXCL12 & Ovarian Serous Adenocarcinoma & -29.039 & -13.783 & 0.000 & [17] \\
\hline CXCL13 & Ovarian Serous Adenocarcinoma & 2.245 & 12.237 & 0.000 & [18] \\
\hline CXCL14 & Ovarian Serous Adenocarcinoma & 2.762 & 6.752 & 0.000 & [19] \\
\hline
\end{tabular}


In addition, we used TCGA data to further verify the level of CXC chemokines expression through the GEPIA database. As expected, we also saw CXCL1, CXCL8, CXCL10, CXCL11, CXCL13, and CXCL14 in tumor tissues were upregulated in the results of TCGA data and the difference was statistically significant $(p<0.05)$. In the TCGA database, only the downregulation of CXCL12 was different, while the expression of CXCL3 was not statistically different (Fig. 2).

Then, we examined the correlation between CXC chemokines and the pathological stage of OC patients and found that the expression of CXCL8 $(P=0.002)$, CXCL11 $(P=0.039)$, and CXCL13 $(P=0.029)$ gradually increased with the aggravation of the pathological stage Decline, showing a trend of negative correlation (Fig. 3A-H). Based on the above results, we seem to have discovered an interesting phenomenon. The expressions of these three CXC chemokines all gradually decrease with the progress of the pathological stage. Further, we analyzed the expression of these chemokines using the data of the TCGA database in the GEPIA database and found that the expression of CXCL10 was the highest and the difference was significant (Fig. 3I).

\section{Analysis of the prognostic value of CXC chemokines in OC patients}

The overall survival curve is shown in Fig. 4. The high expression of CXCL10 $(P=0.004), \operatorname{CXCL11}(P=0.000)$, and CXCL13 $(P=0.003)$ can improve the survival rate of $\mathrm{OC}$ patients. On the other hand, the value of differentially level CXC chemokines in disease-free survival of OC patients was also evaluated. Here, we only found that the high expression of CXCL13 has a promoting effect on disease-free survival (Supplementary Fig. 2).

\section{CXC chemokine expression, co-expression, gene network, and interaction analysis in $O C$ patients}

The temporary data set of TCGA was used to analyze the genetic changes of CXC chemokines. As a result, in the OC samples that were queried, the changes of CXCL1, CXCL3, CXCL8, CXCL10, CXCL11, CXCL12, and CXCL13 were $6,6,6,4,4,4,5$, and $2.9 \%$, respectively (Fig. 5A). Here, amplification accounted for most of the reasons for the change, which also found the reason for the increase of some CXC chemokines. The GeneMANIA database shows that the functions of differentially expressed CXC chemokines were mainly related to cell chemotaxis and chemokine activity related (Fig. 5B). In addition, the results of PPI network analysis of STRING database show that these CXC chemokines interact as shown in the figure (Fig. 5C).

\section{Analysis of GO and pathway of CXC chemokines in OC patients}

First, we obtained 50 genes related to CXC chemokines by referring to previous research results. Then we used Metascape to analyze their functions. Supplementary Fig. 3A shows the top 10 richest GO categories using Metascape. Among the biological process (BP) category, regulation of cytosolic calcium ion concentration pathway, $G$ protein coupled receptor signaling pathway, regulation of secretion, calcium ion transport, and response to wounding were related to the occurrence and development of OC. In the molecular function (MF) category, we found that these genes play a role in both chemokine receptor binding and cytokine receptor binding activity (Supplementary Fig. 3B). Side of membrane, dendritic tree, heterotrimeric G-protein complex, cell body, endocytic vesicle, platelet alpha granule lumen, focal adhesion, vacuum, midbody, and postsynaptic membrane are the 10 most enriched OC categories in cells (Supplementary Fig. 3C). In addition, we also performed KEGG pathway analysis. We found that the chemokine signaling pathways, cytokine-cytokine receptor interactions, and cancer pathways were closely related to tumorigenesis and the progression of OC (Supplementary Fig. 3D).

\section{Transcription factor targets of CXC chemokines in patients with OC}

We use the TRRUST database to explore the transcription factor target information of the differentially expressed CXC chemokines. As shown in Table 2, we found that the transcription factors RELA, SP1, and NFKB1 are involved in the regulation of the biological effects of CXC chemokines. Among them, RELA and NFKB1 have been discovered as key transcription factors of CXCL1, CXCL8, CXCL10, and CXCL12, while SP1 was the key transcription factor of CXCL1 and CXCL14.

\section{Correlation between CXC chemokines and immune cell infiltration in $O C$ patients}

This study used the TIMER database to analyze the correlation between $\mathrm{CXC}$ chemokines and immune cell infiltration. CXCL1 was positively correlated with neutrophils (Cor $=0.275, P<0.05$ ) and dendritic cells (Cor $=0.229, P<0.05$ ). Similarly, CXCL3 was positively correlated with neutrophils (Cor $=0.304, P<0.05$ ) and dendritic cells (Cor $=0.174, P<0.05$ ), in addition to B cells (Cor $=-0.132, P<0.05$ ). There was a negative correlation. CXCL10, CXCL11, and CXCL13 were all positively correlated with B cells, $\mathrm{CD}^{+} \mathrm{T}$ cells, macrophages, neutrophils, and dendritic cells. However, the immune infiltration trend of CXCL12 and CXCL14 was similar. They 
A

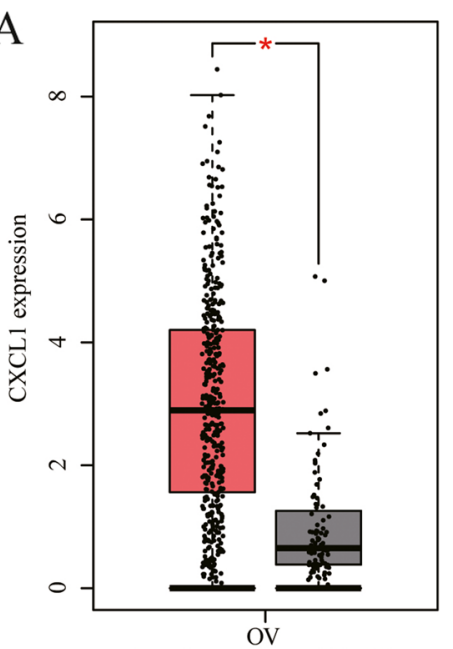

D

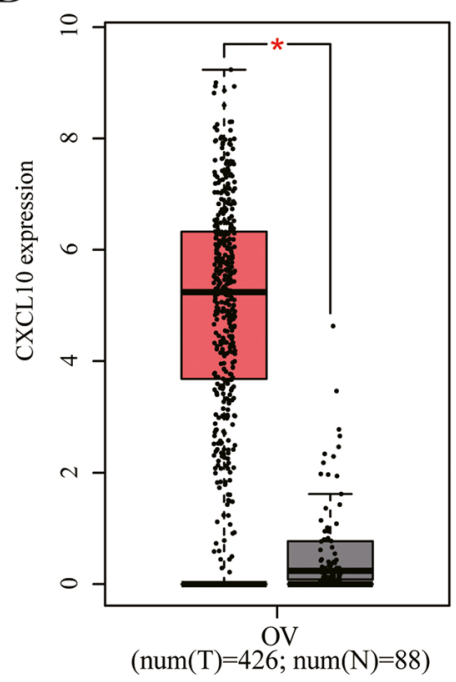

B

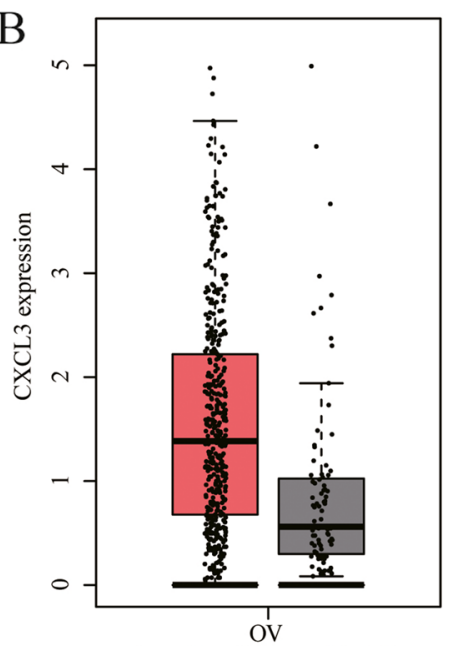

$\mathrm{E}$

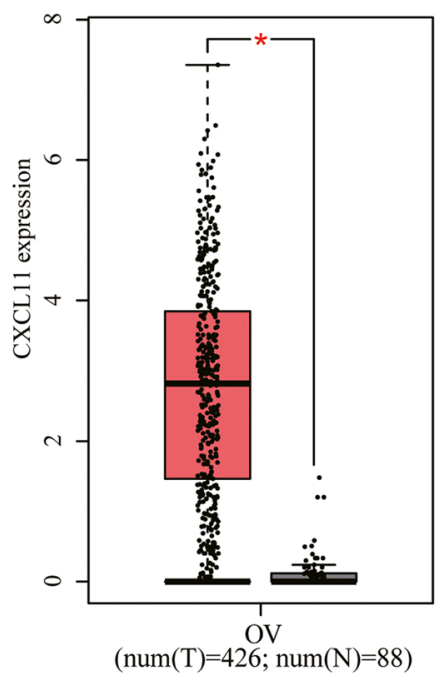

C

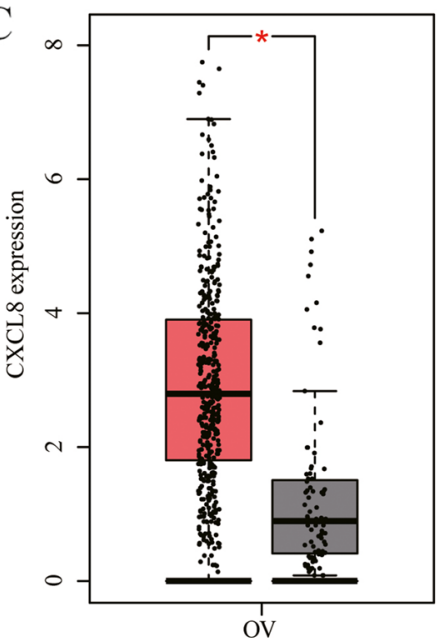

F

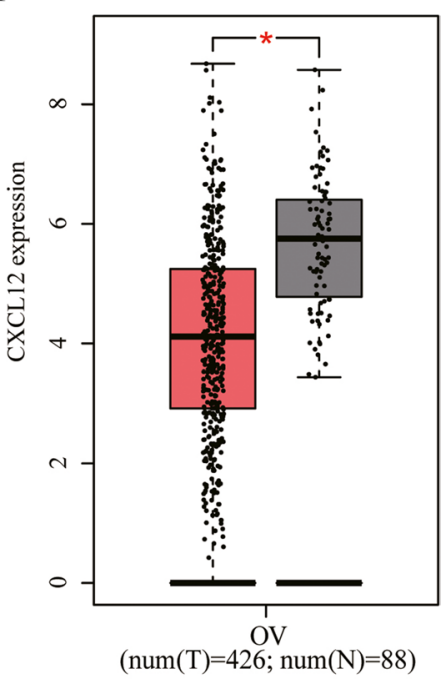

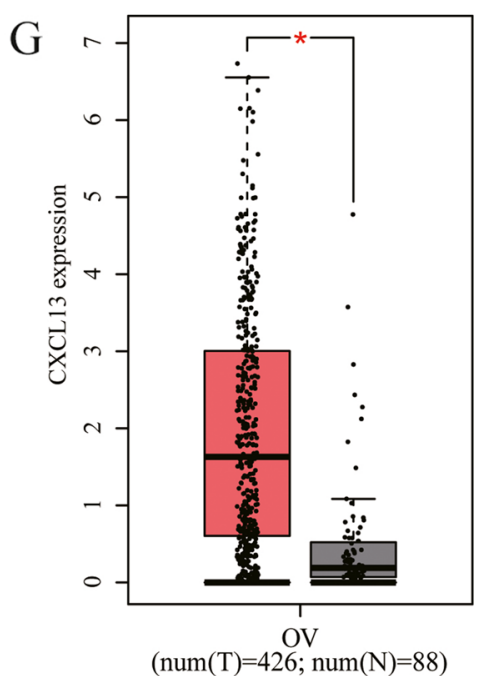

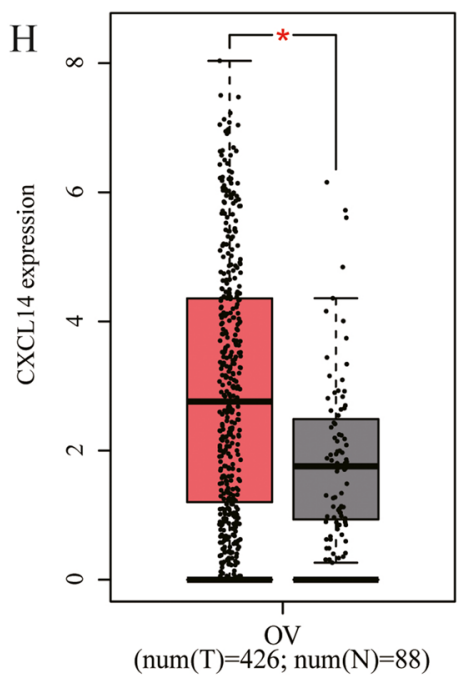

Fig. 2 GEPIA database verifies the expression of CXC chemokines. In this study, the GEPIA database used TCGA data to verify the expression of CXC chemokines. ${ }^{*} p<0.05$ vs. normal 


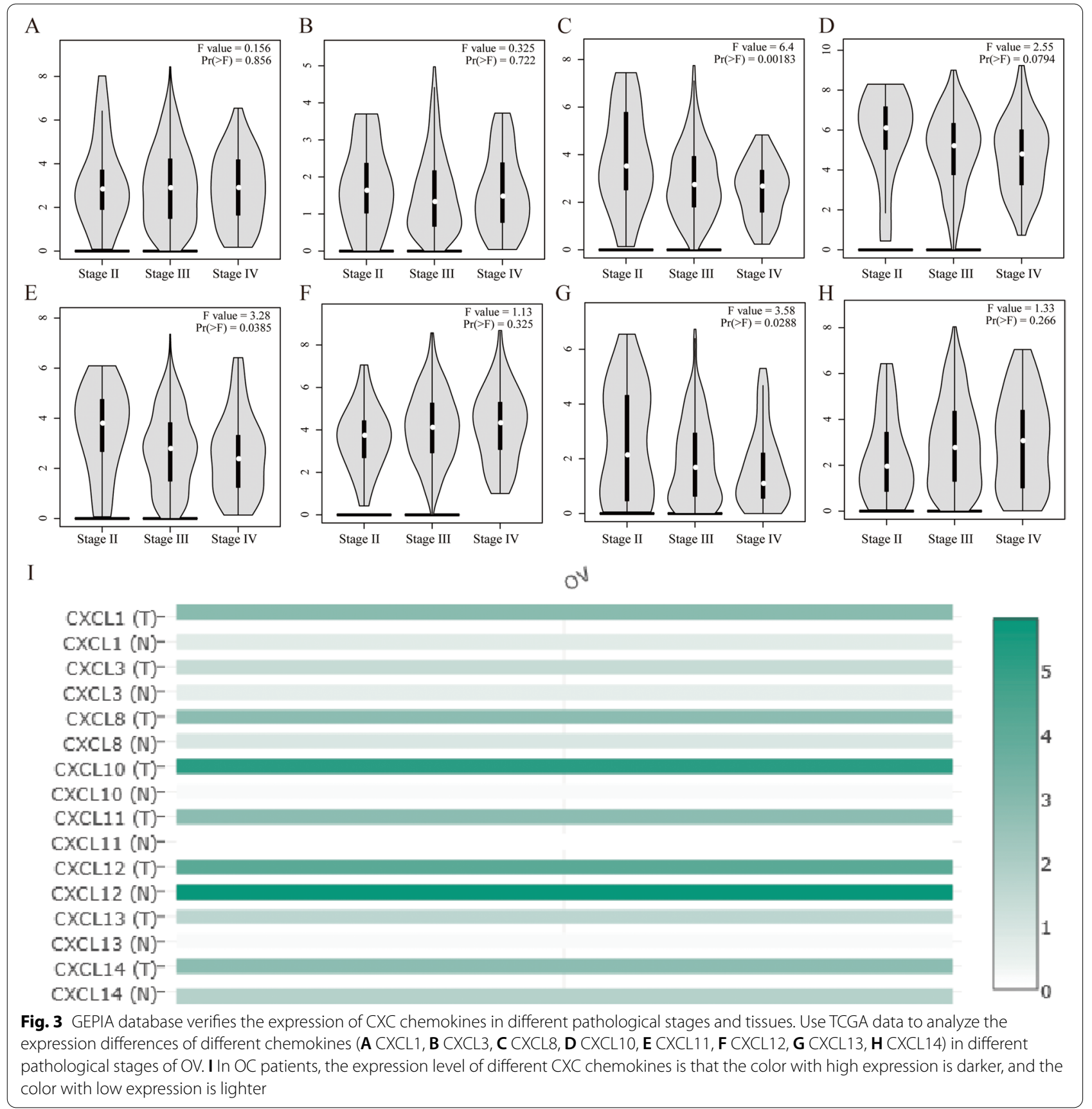

are all positively correlated with macrophages, neutrophils and dendritic cells, but negatively correlated with $\mathrm{B}$ cells (Fig. 6) $(P<0.05)$.

\section{The GEO database detects the expression of CXC chemokines in $\mathrm{OC}$}

Through the above analysis, we have discovered the role of different CXC chemokines in OC. To further verify the expression of different CXC chemokines, we selected the data of two GEO samples of GSE6008 and GSE66957 to verify the expression of them. First, we standardized the data (Fig. 7A-C). After data processing, we also found that the expression of CXCL1, CXCL10, CXCL11, CXCL13, and CXCL14 was significantly increased in tumor tissues, and the difference between CXCL10 was the most significant (Fig. 7D). 

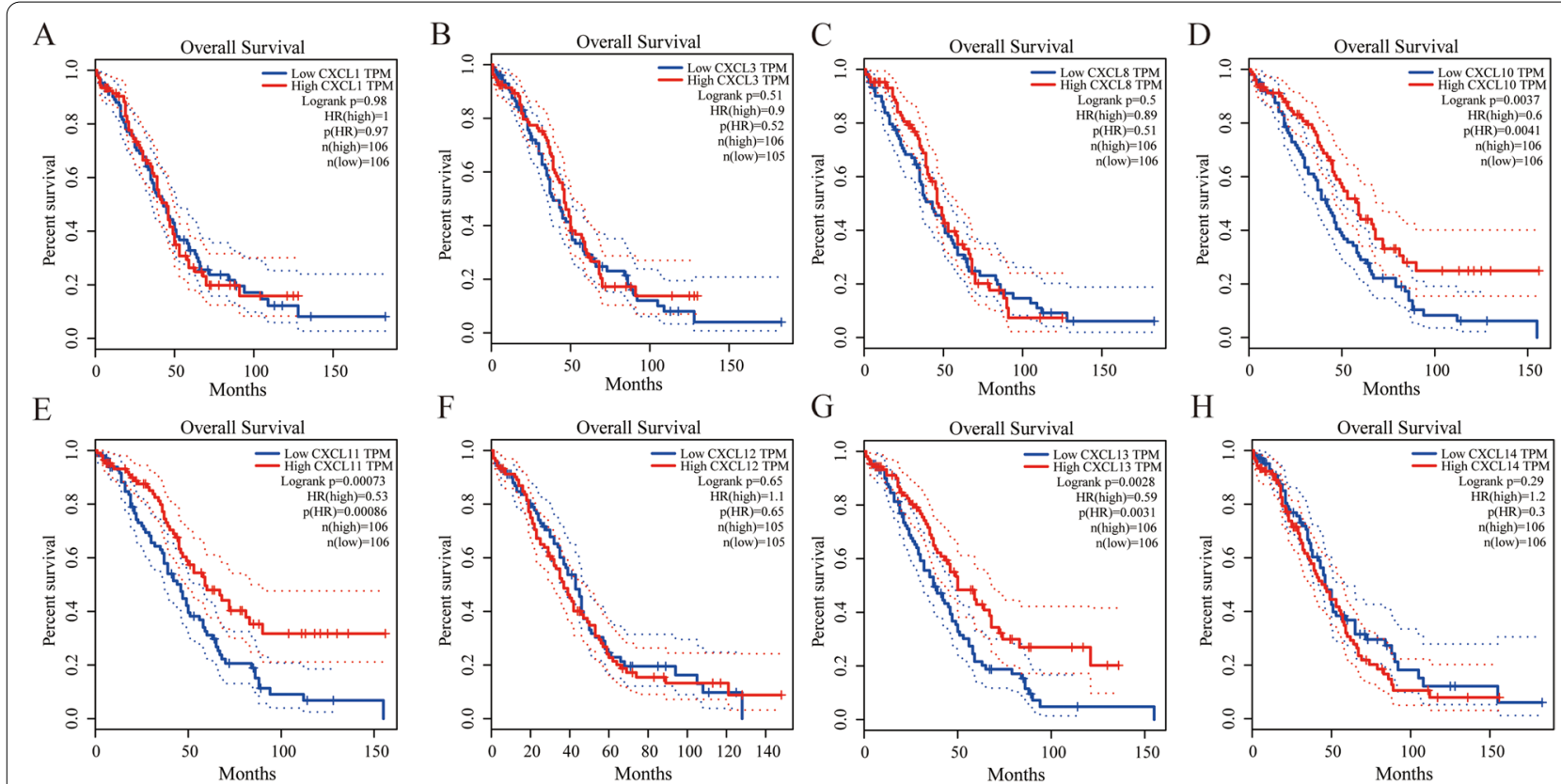

Fig. 4 The prognostic value of different expressed CXC chemokines in OC patients in the overall survival curve. The overall survival curve of $\mathbf{A}$ CXCL1, B CXCL3, C CXCL8, D CXCL10, E CXCL11, F CXCL12, G CXCL13, and H CXCL14 in OC. All survival data are derived from TCGA data in the GEPIA database

\section{The effect of CXCL10 on tumor and angiogenesis}

Through the above bioinformatics analysis, we found that CXCL10 may play an important role in the occurrence and development of OC. In addition, referring to other studies, we found that CXCL10 has a different trend in other tumor studies. In this study, we found that it is correlated with immune infiltration and patient survival. First, we use the $\mathrm{T}$ cell killing test to determine the effect of CXCL10 on immunity. We co-cultured tumor cells with different CXCL10 interference with $\mathrm{T}$ cells and found that overexpression of CXCL10 can effectively enhance tumor suppression and apoptosis caused by $\mathrm{T}$ cells, and this phenomenon was reversed when CXCL10 was inhibited (Fig. 8A-D). Furthermore, we selected an animal model of ovarian cancer that overexpressed CXCL10 for verification. The results showed that the tumor volume and weight after overexpression of CXCL10 were smaller than that of the NC group (Fig. 9A, B) $(P<0.05)$. After further staining the tumor tissue, it was found that the tumor proliferation ability after overexpression of CXCL10 was weakened, and the cell apoptosis was significantly increased (Fig. 9C). Furthermore, after staining the tumor tissue with CD8, we found that the number of CD8 positive cells in the tumor overexpressing CXCL10 was more than that in the NC group. This result shows that CXCL10 can promote the killing effect of cytotoxic $\mathrm{T}$ cells on tumor cells, which is similar to the result of the above analysis (Fig. 9C). Previously shown in other tumors, CXCL10 can effectively inhibit tumor angiogenesis. First of all, when we used differently treated tumor cell culture media to process VE cells to detect angiogenesis, we found that overexpression of CXCL10 could inhibit angiogenesis, while the results of inhibiting CXCL10 are the opposite (Fig. 8E, F). In addition, we further tested angiogenesis markers and immunochemical staining methods to verify this phenomenon (Fig. 9D-F) $(P<0.05)$. In summary, we found that CXCL10 can inhibit the growth of ovarian cancer by increasing immune killing and inhibiting angiogenesis.

\section{Discussion}

A large number of studies had shown that CXC chemokines play an important role in tumorigenesis, proliferation and apoptosis [18]. However, the biological function and prognostic value of CXC chemokines in OC remain unclear. This study was the first to screen CXC chemokine markers in ovarian cancer through bioinformatics methods, and verified through in vivo and in vitro experiments that CXCL10 influences the process of ovarian cancer immune response and angiogenesis. Our data attempts to provide a basis for future ovarian cancer immunotherapy and biomarker selection.

First, we tested the expression of CXC chemokine in tumors and its correlation with the expression in different pathological stages. The results showed that expression of CXCL1, CXCL10, CXCL11, CXCL13, and CXCL14 were 


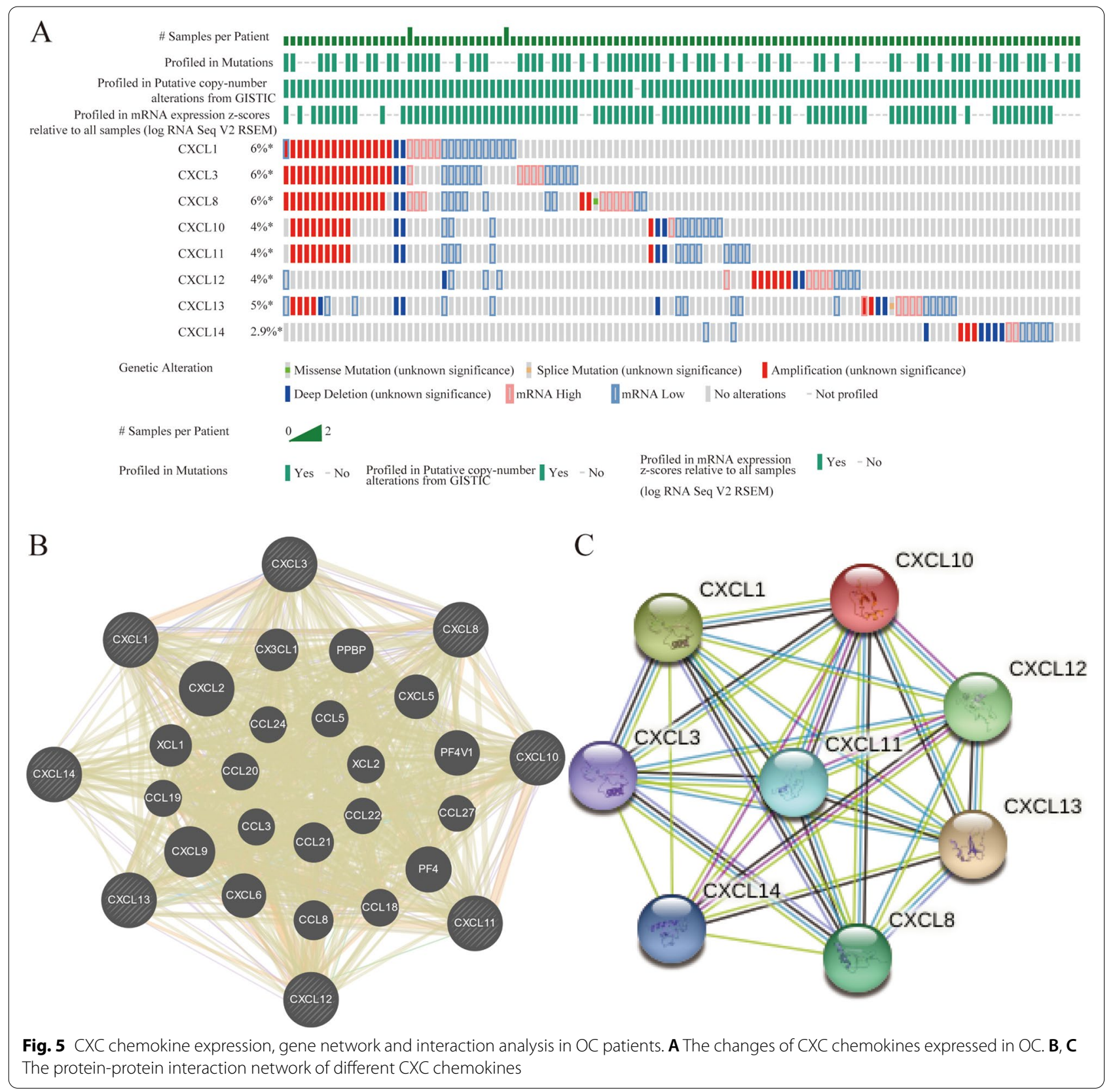

Table 2 Key regulated factor of CXC chemokines in OC (TRRUST)

\begin{tabular}{llllr}
\hline Key TF & Description & Gene & FDR & P value \\
\hline RELA & $\begin{array}{l}\text { v-rel reticuloendotheliosis viral onco- } \\
\text { gene homolog A (avian) }\end{array}$ & CXCL1, CXCL8, CXCL10, CXCL12 & $6.50 \mathrm{E}-06$ \\
NFKB1 & $\begin{array}{l}\text { Nuclear factor of kappa light polypep- } \\
\text { tide gene enhancer in B cells 1 } \\
\text { Sp1 transcription factor }\end{array}$ & CXCL1, CXCL8, CXCL10, CXCL12 & $6.50 \mathrm{E}-06$ \\
SP1 & CXCL1, CXCL14 & 0.0158 & $4.33 \mathrm{E}-06$ \\
\hline
\end{tabular}




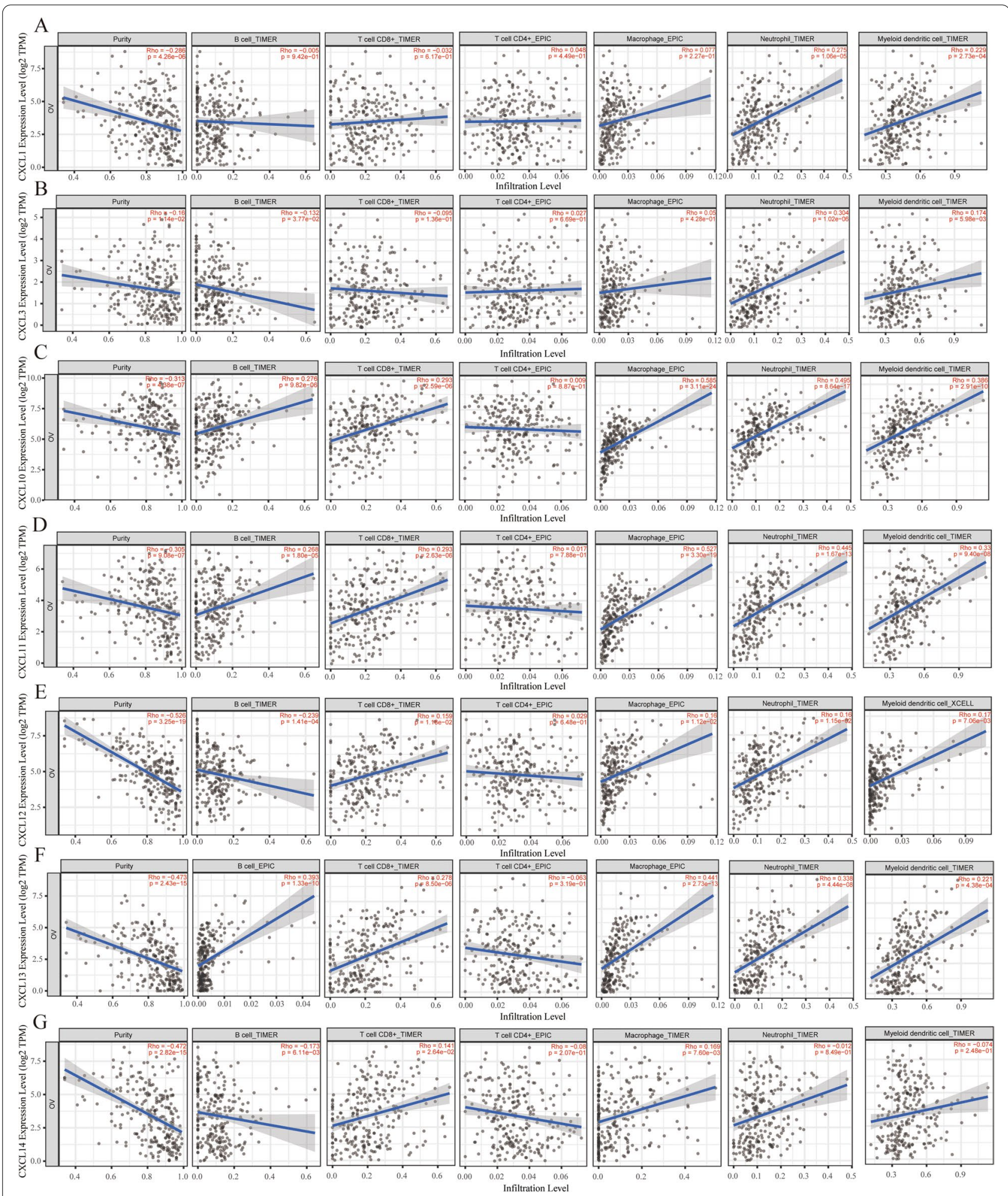

Fig. 6 Correlation between $C X C$ chemokines and immune cell infiltration in OC patients. The correlation between the abundance of immune cell and the expression of A CXCL1, B CXCL3, C CXCL8, D CXCL10, E CXCL11, F CXCL12, G CXCL13, and H CXCL14 in OC 


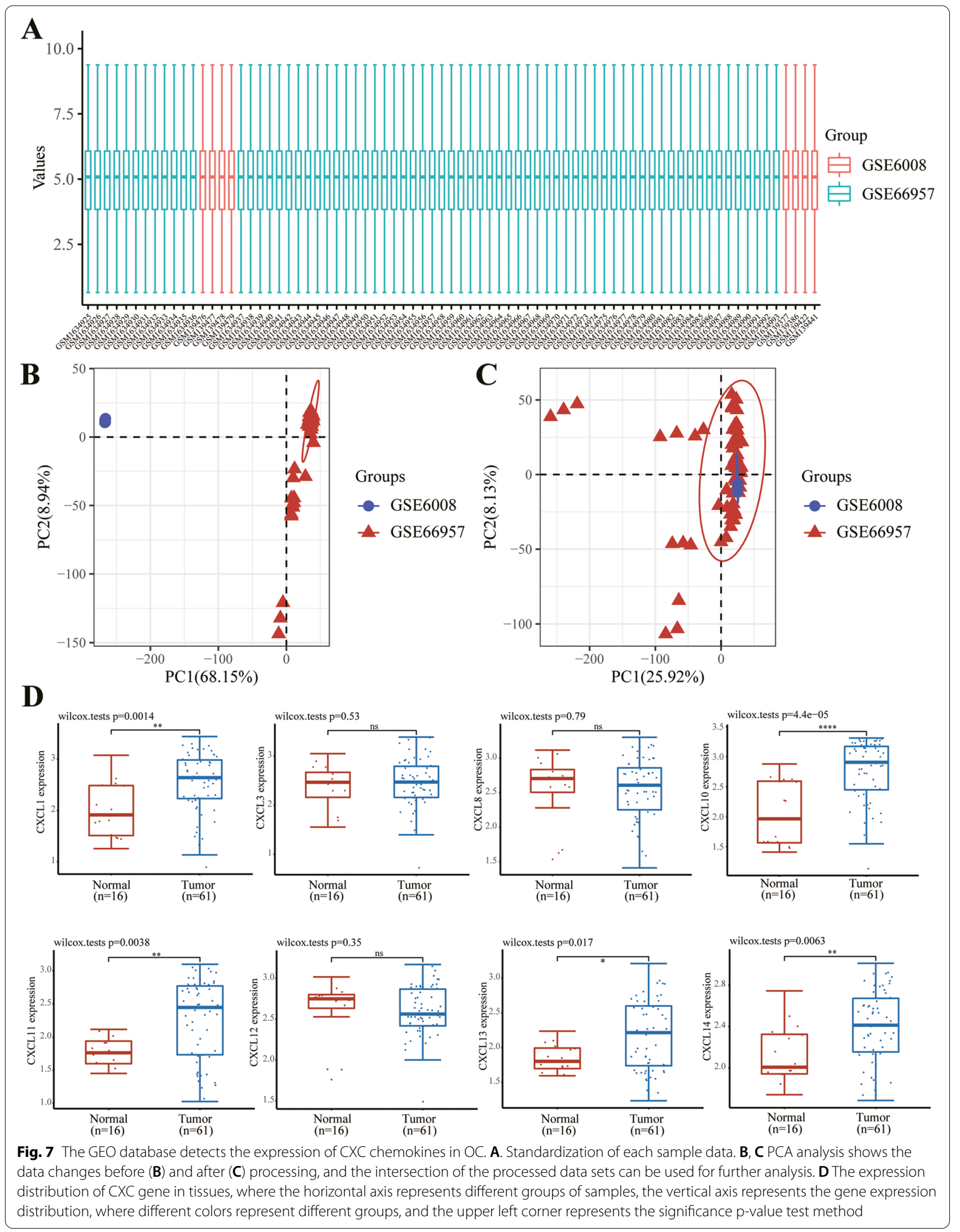




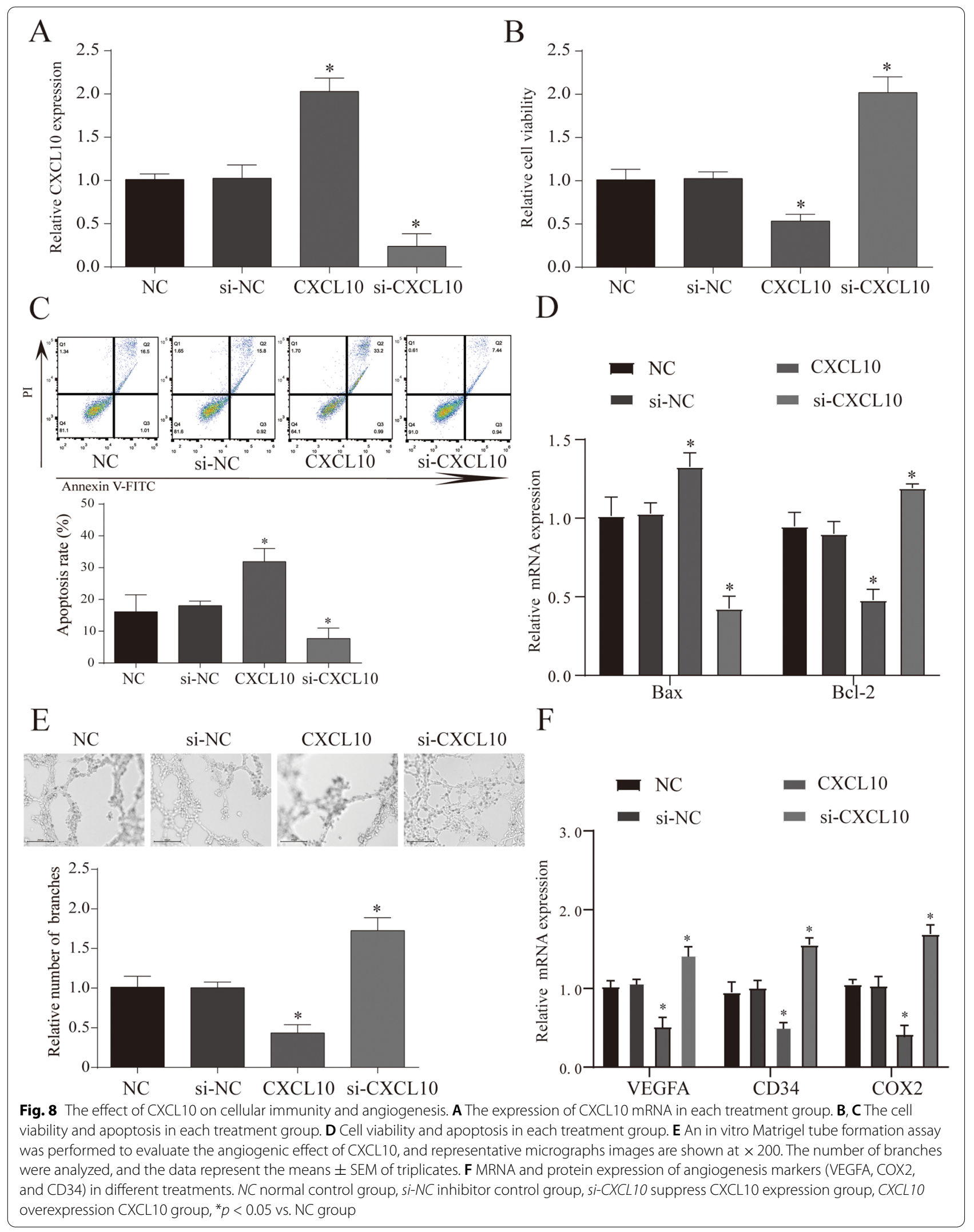



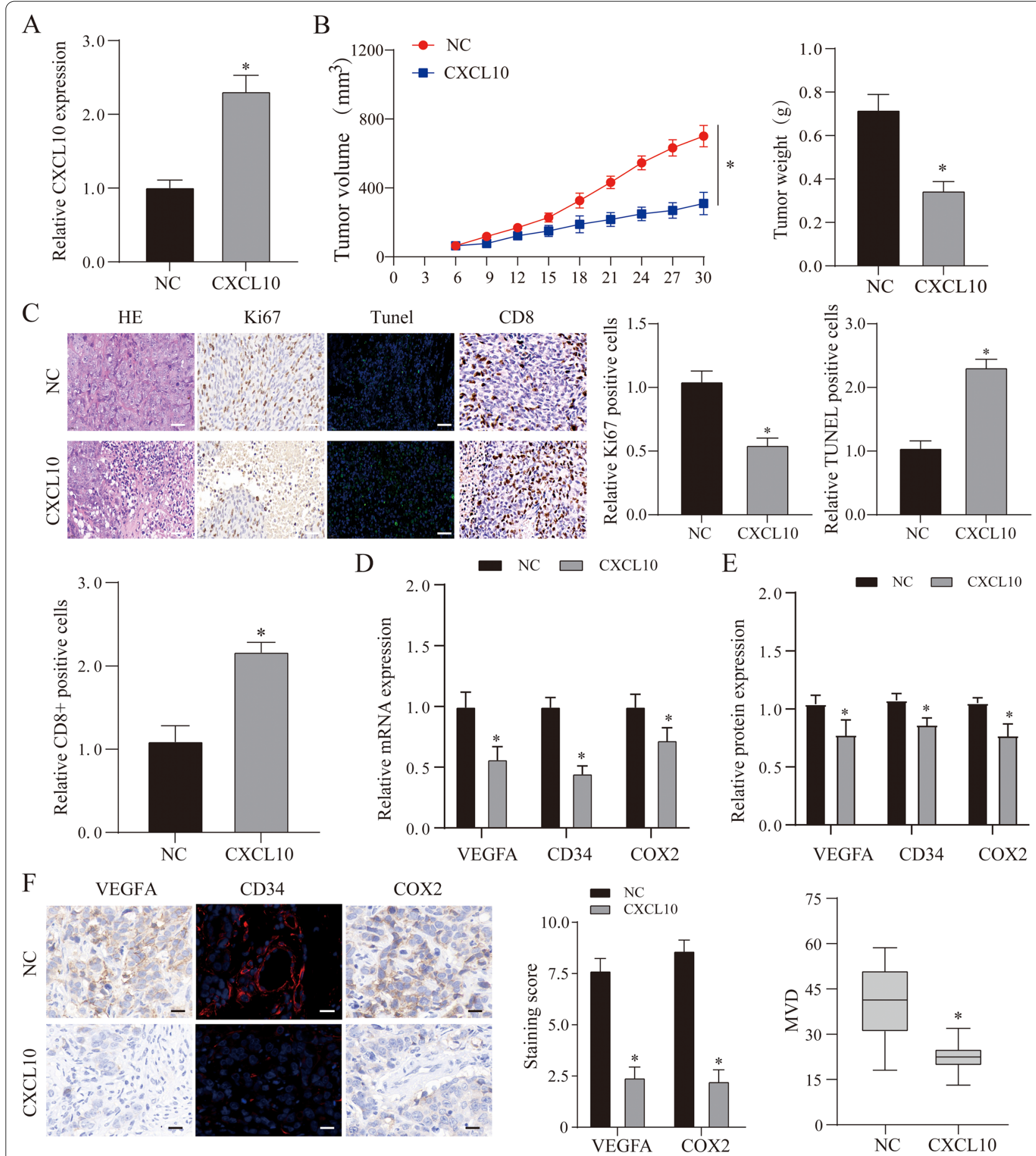

Fig. 9 The effect of CXCL10 on tumor and angiogenesis. A QRT-PCR verified the expression of CXCL10 mRNA. B Tumor size and volume in each treatment group. C Tumor tissue HE, KI67, TUNEL, and CD8 ${ }^{+}$cell staining results. We use Image J to quantitatively analyze the number of KI67, TUNEL, and CD8-positive cells, and all data are expressed in the form of mean + SEM. D, E MRNA and protein expression of angiogenesis markers (VEGFA, COX2, and CD34) in different treatments. $\mathbf{F} I H C$ scores of COX2 and VEGFA in tissues was compared with each group. MVD was calculated as the average measurement of 10 random high-power fields (20x). NC normal control group, si-NC inhibitor control group, si-CXCL10 suppress CXCL10 expression group, CXCL10 overexpression CXCL10 group, ${ }^{*} p<0.05$ vs. NC group 
significantly increased in $\mathrm{OC}$ tissues. These results are obtained by re-arguing with two GEO database samples. They were similar to the results of previous literatures. In addition, we found that increased CXCL10, CXCL11, and CXCL13 expression increased survival in patients with $\mathrm{OC}$ with tumor progression. These data suggest that they may play an important role in the OC process. Although the effect of CXC chemokine on ovarian cancer has been reported before [19], the specific role and mechanism of CXC chemokine are still unclear.

Due to the phenomenon of differential expression of CXC chemokines, we conducted a comprehensive analysis on the molecular characteristics of differential expression of CXC chemokines. The results showed that real amplification was the main reason for this change, which also found the reason for the increase of some CXC chemokines. Tumorigenesis is complex and multifaceted, and genetic changes play an important role in this process. Zeng et al's study found a synergistic effect of CXC chemokines in renal cancer [18].

Then, we used the Metascape database to perform GO and KEGG pathway enrichment analysis on the verified differentially expressed CXC chemokines to determine their possible biological functions. Through the above data, we confirmed that these genes may function through chemokine signaling pathways, cytokinecytokine receptor interactions and other pathways. A lot of reports had shown that the chemokine signaling pathways may play an important role in the proliferation, aging, angiogenesis, and immune escape of cancers. This also provides us with further molecular biology experiments. In summary, the differential expression of CXC chemokines may be a potential drug target for the treatment of OC patients.

Rela phosphorylation has been shown to be involved in disease progression, particularly in inflammatory disease and cancer, by regulating NF- $\kappa B$ signal pathway [22]. However, NFKB1 is an inhibitor of inflammation and cancer and plays a tumor-inhibiting role by reducing abnormal activation of the NF- $\kappa B$ signaling pathway [23]. The role of SP1 in ovarian cancer has been discovered by people. For example, Zhang et al. found that SP1-12LOX axis plays a role in the progression and drug resistance of ovarian cancer [24]. Other studies have shown that it can affect the progression of ovarian cancer by affecting the generation of long non-coding RNA [25]. These data support the interpretation of the effect of CXC chemokines in OC.

There is increasing evidence that immune cell infiltration can influence tumor progression and recurrence and become an important determinant of immunotherapeutic response and clinical outcome
[26].CD4 ${ }^{+} \mathrm{T}$ cells recognize cancer antigens, while macrophages may be involved in tumor inhibition [27]. In this study, we found that the level of CXC chemokines was related to the infiltration of immune cells. The correlation indicates that they reflect the effective tumor immune status and provide data for immunotherapy.

Finally, we chose two GEO data (GSE6008 and GSE66957) to try to further study different CXC chemokines. According to the multiples of differential expression, the expression level of tumor tissues, and the importance of biological functions in tumors, CXCL10 was selected for in-depth discussion. The study found that CXCL10 could inhibit tumor growth by constructing a model of ovarian cancer with overexpression of CXCL10. As previously reported, CXC10 plays a different role in different types of tumors [28]. As we predicted using the database, we first verified the immune effect of CXCL10 through T cell killing experiments and found that overexpression of CXCL10 can promote the killing of tumor cells by $\mathrm{T}$ cells. Furthermore, we used immunohistochemistry to detect the overexpression of CXCL10 in tumor models and found that overexpression can cause more cytotoxic $\mathrm{T}$ cells to infiltrate the tumor tissue.

This study is the first to demonstrate its ability to inhibit tumor growth in ovarian cancer. Further, some studies have found that CXCL10 can exert tumor inhibition by inhibiting angiogenesis in colorectal cancer, lung cancer, cervical cancer, and other tumors [29,30]. Therefore, we selected CD34, COX2, and VEGFA to detect the markers of angiogenesis, and found that the overexpression of CXCL10 in OC tissue could effectively inhibit angiogenesis. This phenomenon has been further verified by the angiogenesis experiment of VE cells treated with the culture medium after in vitro tumor cell treatment. This may be one of the reasons for its tumor-inhibiting effect.

This study was the first to explore the expression and biological functions of a variety of CXC chemokines in OC. Through in-depth bioinformatics analysis, we selected CXCL10 as the object of further research. We also verified the role of CXCL10 in the OC process. OC is currently one of the deadliest gynecological malignancies. Surgery and chemotherapy are the main treatments for ovarian cancer. In the past two decades, immunotherapy has developed rapidly [31]. In the future, this approach may change the main development direction of various cancer therapies. However, the current immunotherapy response rate for ovarian cancer patients is still moderate, but with the development of new treatments such as TCR engineered T cells, this situation will be changed [32]. 


\section{Conclusions}

Our results are the first to find that CXCL10 can not only activate the killing effect of $\mathrm{T}$ cells in the body on tumors, but also we have seen its impact on angiogenesis on the other hand. Current studies have found that using immunotherapy as an adjuvant therapy, combined with anti-angiogenic drugs, can significantly improve the efficiency of treatment. This view fits the conclusion of this research. Nevertheless, we still lack clinical sample data and in-depth discussion of its mechanism, which will be one of the main directions of our future research. In short, our data discovered the effects of different CXC chemokines on ovarian cancer and conducted in-depth verification of CXCL10 in two aspects: immune regulation and angiogenesis. This study will provide novel insights for the selection of immunotherapy targets and prognostic markers for $\mathrm{OC}$ patients.

\section{Abbreviations}

OC: Ovarian cancer; GO: Gene ontology; KEGG: Kyoto Encyclopedia of Genes and Genome; TF: Transcription factor; HE: Hematoxylin-eosin; WB: Western blot; OV: Ovarian serous cystadenocarcinoma.

\section{Supplementary Information}

The online version contains supplementary material available at https://doi. org/10.1186/s12957-021-02440-x.

\section{Additional file 1: Supplementary table 1. Primer sequence.}

Additional file 2: Supplementary Figure 1. Oncomine database analysis of the expression of different chemokines. Supplementary Figure 2 . The prognostic value of different expressed CXC chemokines in OC patients in the disease free survival curve. Supplementary Figure $3 \mathrm{GO}$ and pathway analysis of CXC chemokines related genes using Metascape database.

\section{Acknowledgements}

None.

\section{Authors' contributions}

W.Y.L. conceived and designed research. W.Y.L. performed experiments. W.Y.L and J.A.M interpreted results of experiments. W.Y.L and S.X. analyzed data and prepared figures. C.J.X. drafted paper. W.Y.L. edited and revised manuscript. All authors read and approved final version of manuscript.

\section{Funding}

This work was supported by the National Natural Science Foundation of China (Grant No.81860499).

\section{Availability of data and materials}

All data generated or analyzed during this study are included in this published article.

\section{Declarations}

Ethics approval and consent to participate

All experimental protocols in this study were approved by the Ethics Committee of Yunnan University (No.YNU20210121).

\section{Consent for publication}

Not applicable.

\section{Competing interests}

The authors declare no competing interests.

Received: 19 August 2021 Accepted: 2 November 2021

Published online: 18 November 2021

\section{References}

1. Forstner R. Early detection of ovarian cancer. Eur Radiol. 2020;30(10):5370-3.

2. Rådestad E, Klynning C, Stikvoort A, et al. Immune profiling and identification of prognostic immune-related risk factors in human ovarian cancer. Oncoimmunology. 2019;8(2):e1535730.

3. Song $M$, Yeku OO, Rafiq S, et al. Tumor derived UBR5 promotes ovarian cancer growth and metastasis through inducing immunosuppressive macrophages. Nat Commun. 2020;11(1):6298.

4. Tokunaga R, Zhang W, Naseem M, et al. CXCL9, CXCL10, CXCL11/CXCR3 axis for immune activation-a target for novel cancer therapy. Cancer Treat Rev. 2018:63:40-7.

5. Bronger $\mathrm{H}$, Singer J, Windmüller $\mathrm{C}$, et al. CXCL9 and CXCL10 predict survival and are regulated by cyclooxygenase inhibition in advanced serous ovarian cancer. Br J Cancer. 2016;115(5):553-63.

6. Au KK, Peterson N, Truesdell P, et al. CXCL10 alters the tumour immune microenvironment and disease progression in a syngeneic murine model of high-grade serous ovarian cancer. Gynecol Oncol. 2017;145(3):436-45.

7. Rhodes DR, Yu J, Shanker K, et al. ONCOMINE: a cancer microarray database and integrated data-mining platform. Neoplasia. 2004;6(1):1-6.

8. Wu P, Heins ZJ, Muller JT, et al. Integration and analysis of CPTAC proteomics data in the context of cancer genomics in the cBioPortal. Mol Cell Proteomics. 2019:18(9):1893-8.

9. Franz M, Rodriguez H, Lopes C, et al. GeneMANIA update 2018. Nucleic Acids Res. 2018;46(W1):W60-W4.

10. Zhou Y, Zhou B, Pache L, et al. Metascape provides a biologist-oriented resource for the analysis of systems-level datasets. Nat Commun. 2019;10(1):1523.

11. Han $\mathrm{H}$, Cho JW, Lee S, et al. TRRUST v2: an expanded reference database of human and mouse transcriptional regulatory interactions. Nucleic Acids Res. 2018;46(D1):D380-D6.

12. LiT, Fu J, Zeng Z, et al. TIMER2.0 for analysis of tumor-infiltrating immune cells. Nucleic Acids Res. 2020;48(W1):W509-W14.

13. Lim SO, Li CW, Xia W, et al. Deubiquitination and Stabilization of PD-L1 by CSN5. Cancer Cell. 2016;30(6):925-39.

14. Lu KH, Patterson AP, Wang $L$, et al. Selection of potential markers for epithelial ovarian cancer with gene expression arrays and recursive descent partition analysis. Clin Cancer Res. 2004;10(10):3291-300.

15. Yoshihara K, Tajima A, Komata D, et al. Gene expression profiling of advanced-stage serous ovarian cancers distinguishes novel subclasses and implicates ZEB2 in tumor progression and prognosis. Cancer Sci. 2009;100(8):1421-8.

16. Bonome T, Levine DA, Shih J, et al. A gene signature predicting for survival in suboptimally debulked patients with ovarian cancer. Cancer Res. 2008;68(13):5478-86

17. Hendrix ND, Wu R, Kuick R, et al. Fibroblast growth factor 9 has oncogenic activity and is a downstream target of Wnt signaling in ovarian endometrioid adenocarcinomas. Cancer Res. 2006;66(3):1354-62.

18. Zeng Q, Sun S, Li Y, et al. Identification of therapeutic targets and prognostic biomarkers among CXC chemokines in the renal cell carcinoma microenvironment. Front Oncol. 2019;9:1555.

19. Zeng Y, Li B, Liang Y, et al. Dual blockade of CXCL12-CXCR4 and PD-1-PDL1 pathways prolongs survival of ovarian tumor-bearing mice by prevention of immunosuppression in the tumor microenvironment. FASEB J. 2019;33(5):6596-608

20. Adib TR, Henderson S, Perrett $C$, et al. Predicting biomarkers for ovarian cancer using gene-expression microarrays. Br J Cancer. 2004:90(3):686-92.

21. Welsh JB, Zarrinkar PP, Sapinoso LM, et al. Analysis of gene expression profiles in normal and neoplastic ovarian tissue samples identifies candidate molecular markers of epithelial ovarian cancer. Proc Natl Acad Sci U S A. 2001;98(3):1176-81. 
22. Lu X, Yarbrough WG. Negative regulation of RelA phosphorylation: emerging players and their roles in cancer. Cytokine Growth Factor Rev. 2015;26(1):7-13.

23. Didonato JA, Mercurio F, Karin M. NF-kappaB and the link between inflammation and cancer. Immunol Rev. 2012;246(1):379-400.

24. Zhang Q, Yan G, Lei J, et al. The SP1-12LOX axis promotes chemoresistance and metastasis of ovarian cancer. Mol Med. 2020;26(1):39.

25. Lang C, Dai Y, Wu Z, et al. SMAD3/SP1 complex-mediated constitutive active loop between IncRNA PCAT7 and TGF-beta signaling promotes prostate cancer bone metastasis. Mol Oncol. 2020;14(4):808-28.

26. Bindea G, Mlecnik B, Tosolini M, et al. Spatiotemporal dynamics of intratumoral immune cells reveal the immune landscape in human cancer. Immunity. 2013;39(4):782-95.

27. Lin P, Guo YN, Shi L, et al. Development of a prognostic index based on an immunogenomic landscape analysis of papillary thyroid cancer. Aging (Albany NY). 2019;11(2):480-500.

28. Bagheri H, Pourhanifeh MH, Derakhshan M, et al. CXCL-10: a new candidate for melanoma therapy? Cell Oncol (Dordr). 2020;43(3):353-65.
29. Li G, Tian L, Hou JM, et al. Improved therapeutic effectiveness by combining recombinant CXC chemokine ligand 10 with Cisplatin in solid tumors. Clin Cancer Res. 2005;11(11):4217-24.

30. Nagpal ML, Davis J, Lin T. Overexpression of CXCL10 in human prostate LNCaP cells activates its receptor (CXCR3) expression and inhibits cell proliferation. Biochim Biophys Acta. 2006;1762(9):811-8.

31. Yang C, Xia BR, Zhang ZC, et al. Immunotherapy for ovarian cancer: adjuvant, combination, and neoadjuvant. Front Immunol. 2020;11:577869.

32. Odunsi K. Immunotherapy in ovarian cancer. Ann Oncol. 2017;28(suppl_8):viii1-7.

\section{Publisher's Note}

Springer Nature remains neutral with regard to jurisdictional claims in published maps and institutional affiliations.
Ready to submit your research? Choose BMC and benefit from:

- fast, convenient online submission

- thorough peer review by experienced researchers in your field

- rapid publication on acceptance

- support for research data, including large and complex data types

- gold Open Access which fosters wider collaboration and increased citations

- maximum visibility for your research: over $100 \mathrm{M}$ website views per year

At BMC, research is always in progress.

Learn more biomedcentral.com/submissions 